\title{
Conservation phylogeography: does historical diversity contribute to regional vulnerability in European tree frogs (Hyla arborea)?
}

\author{
CHRISTOPHE DUFRESNES, * JÉRÔME WASSEF, * KARIM GHALI, ${ }^{\dagger}$ ALAN BRELSFORD,* \\ MATTHIAS STÖCK,*+PETROS LYMBERAKIS, § JELKA CRNOBRNJA-ISAILOVIC ${ }^{* *}$ and \\ NICOLAS PERRIN* \\ *Department of Ecology \& Evolution, University of Lausanne, Biophore Building, 1015 Lausanne, Switzerland, †Vivarium of \\ Lausanne, Ch. De Boissonnet 82, 1010 Lausanne, Switzerland, \$Leibniz-Institute of Freshwater Ecology and Inland Fisheries \\ (IGB), Müggelseedamm 301, D-12587 Berlin, Germany, §Natural History Museum of Crete, University of Crete, Knosos Av., \\ P.O. Box 2208, 71409 Irakleio, Crete, Greece, $\mid$ Faculty of Sciences and Mathematics, University of Niš, Višegradska 33, 18000 \\ Niš, Serbia, **Institute for biological research "S. Stanković", University of Belgrade, Despota Stefana 142, 11000 Beograd, \\ Serbia
}

\begin{abstract}
Documenting and preserving the genetic diversity of populations, which conditions their long-term survival, have become a major issue in conservation biology. The loss of diversity often documented in declining populations is usually assumed to result from human disturbances; however, historical biogeographic events, otherwise known to strongly impact diversity, are rarely considered in this context. We apply a multilocus phylogeographic study to investigate the late-Quaternary history of a tree frog (Hyla arborea) with declining populations in the northern and western part of its distribution range. Mitochondrial and nuclear polymorphisms reveal high genetic diversity in the Balkan Peninsula, with a spatial structure moulded by the last glaciations. While two of the main refugial lineages remained limited to the Balkans (Adriatic coast, southern Balkans), a third one expanded to recolonize Northern and Western Europe, loosing much of its diversity in the process. Our findings show that mobile and $a$ priori homogeneous taxa may also display substructure within glacial refugia ('refugia within refugia') and emphasize the importance of the Balkans as a major European biodiversity centre. Moreover, the distribution of diversity roughly coincides with regional conservation situations, consistent with the idea that historically impoverished genetic diversity may interact with anthropogenic disturbances, and increase the vulnerability of populations. Phylogeographic models seem important to fully appreciate the risks of local declines and inform conservation strategies.
\end{abstract}

Keywords: biodiversity, conservation genetics, Hyla arborea, phylogeography, Quaternary refugia, red list status

Received 27 November 2012; revision received 24 August 2013; accepted 28 August 2013

\section{Introduction}

Documenting and maintaining the genetic diversity of populations are becoming a central issue in conservation biology (Beebee 2005; Hughes et al. 2008). A link

Correspondence: Nicolas Perrin, Fax: +41(0)21 69241 65;

E-mail: Nicolas.Perrin@unil.ch between diversity and viability has been established across many taxonomic groups (e.g. Oostermeijer et al. 1995; Rowe et al. 1999; Luijten et al. 2000; Hansson \& Westerberg 2002; Reed \& Frankham 2003), and genetic erosion is thought to be a major player of extinction vortices (e.g. Newman \& Pilson 1997; Saccheri et al. 1998; Westemeier et al. 1998; Rowe \& Beebee 2003; Frankham 2005). In addition, intraspecific variability 
represents an adaptive potential to face sudden environmental changes and conditions the capacity of populations to colonize and survive in suboptimal habitats (e.g. Meagher 1999; Frankham 2005; Rowe \& Beebee 2005). As a consequence, conservation measures are designed to maximize the amount of protected diversity, especially through the management of evolutionary significant units (ESU, 'phylogeographic' approach, Moritz 1994) or evolutionary populations ('demographic' approach, Waples \& Gaggiotti 2006).

Anthropogenic factors, such as land use or climate change, are often proposed as the main triggers for the decrease in biodiversity worldwide (e.g. Vitousek et al. 1997; Epps et al. 2005). Especially, genetic impoverishment is usually assumed to result from population disconnection in fragmented landscapes (van Dongen et al. 1998; Buza et al. 2000; Madsen et al. 2000). However, Quaternary climatic oscillations have already strongly influenced the distribution of genetic variation (Hewitt 2000). Formerly, glaciated regions are expected to feature shallow genetic structure, with poor intraspecific variation, as a result of multiple bottlenecks during post-glacial expansions from a single or a few source population(s) (Hewitt 2000). In contrast, refugial areas that survived several northern glaciations constitute hotspots of diversity (Petit et al. 2003), because of both a higher demographic stability and more pronounced structure due to allopatric differentiation ('refugia within refugia', Gómez \& Lunt 2007). As a consequence, ancient and genetically rich southern populations are expected to be in better condition to withstand anthropogenic factors than are recently expanded and genetically impoverished northern populations (Schmitt 2007), a prediction that has rarely been tested (Schmitt \& Hewitt 2004). Species with wide distributions and regional information on their conservation status are best suited to address this fundamental question.

The European tree frog (Hyla arborea) recently expanded from the Balkan Peninsula, from which it recolonized most parts of Central and North-Western Europe (Stöck et al. 2012). Interestingly, its conservation situation across the range is much contrasted: while the species is not considered threatened in south-eastern Europe, it is mostly declining and regionally endangered in northern and western ranges (Fig. S1 and Table S1, Supporting information). A recent study by Luquet et al. (2011) evidenced a positive correlation between heterozygosity and fitness in tree frogs, highlighting the importance of genetic diversity for population viability. In line, several population genetics surveys independently documented low levels of variability in Western Europe, where $H$. arborea is the most vulnerable (Edenhamn et al. 2000; Andersen et al. 2004; Arens et al. 2006; Dubey et al. 2009). Although this was attributed to population bottlenecks associated with landscape management, these studies did not recover signs of disconnection within metapopulations. Alternatively, we propose that historical expansions from southern refugia could account for this reduced diversity and potentially increase the susceptibility of Western European populations to the current anthropogenic pressures responsible for their severe decline (especially industrial agriculture, Brühl et al. 2013).

From previous phylogenetic and phylogeographic studies (Stöck et al. 2008b, 2012), H. arborea forms a genetically poor monophyletic clade with little variation across its range, suggesting one uniform glacial refugium with a global post-glacial expansion. Nevertheless, cytochrome $b$ networks identified a few slightly diverged haplotypes in some of the southern localities (Stöck et al. 2012; see also Stöck et al. 2008b for nuclear data), potentially indicative of cryptic structure. In this study, we use a higher-resolution framework, with fasterevolving molecular markers and denser sampling, (i) to test whether the vulnerable condition of Western European $H$. arborea populations is consistent with a biogeographic loss of variability and (ii) to search for possible cryptic structures associated with refugia and re-expansions during and after the last glaciations, asking in particular whether recently diverged populations are relevant for defining conservation units.

\section{Methods}

\section{DNA extraction}

A total of 779 specimens from 65 localities (considered as separated populations) covering the entire distribution range of $H$. arborea were included in this study (Table S2 and S3, Supporting information). Genomic DNA was extracted from ethanol-preserved tissues (tadpole tail-tips, adult vouchers) and noninvasive buccal swabs (live adults; Broquet et al. 2007) with the Qiagen DNeasy Tissue kit or the Qiagen BioSprint robotic workstation.

\section{Sequence data}

Two mitochondrial and one nuclear marker were sequenced in representative subsets of samples. Detailed protocols and primer information are provided in Data S1 and Table S4 (Supporting information). Following Stöck et al. (2008b, 2012), we first amplified the mitochondrial gene cytochrome $b$ (957 bp) in 211 new samples, complemented by 27 cyt $b$ sequences from Stöck et al. (2012). Second, we developed new primers (adapted from Goebel et al. 1999) to sequence $590 \mathrm{bp}$ from the $5^{\prime}$ hypervariable region of the mitochondrial 
D-loop (Table S4, Supporting information) in these 238 individuals. In addition, we sequenced the D-loop in two $H$. orientalis samples for which cyt $b$ was already available. Mitochondrial sequences could then be concatenated (238 H. arborea samples representing 59 localities, + two individuals of $H$. orientalis). Third, based on the alignment of sequences available on GENBANK, new primers were designed to amplify a portion of the nuclear gene rag-1 (737 bp; Table S4, Supporting information) in 100 individuals. This was complemented by eight published sequences (Stöck et al. 2008b, 2012), for a total of $108 \mathrm{H}$. arborea (55 localities), and we also included rag-1 sequences from closely related hylid species. All sequences were visualized on an ABI-3730 sequencer (AppliedBiosystems, INC.) and aligned using SEAVIEW 4.2 (Gouy et al. 2010). For rag-1, haplotypes of heterozygous individuals were reconstructed with the phase algorithm implemented in DNASP 5 (Librado \& Rozas 2009), using a recombination model with no assumption about rate variation and an initial estimate of 0.0004 . The MCMC chain was run for 1000 iterations with a burnin of 100 and a thinning interval of 1 , and output probability thresholds were set to 0.9 .

\section{Microsatellite data}

Seventeen previously published autosomal microsatellites (Arens et al. 2000; Berset-Brändli et al. 2008a,b) were used in this study. We also included three new autosomal microsatellite loci recently developed on the basis of transcriptomic sequences from H. arborea (Brelsford et al. 2013) and took advantage of this transcriptome to develop ten additional polymorphic microsatellites, following the exact same methodology. Altogether, 750 individuals from 53 populations were genotyped for these 30 markers (see Data S1 and Table S4, Supporting information for information on protocols and primers). In most cases, PCRs were performed in multiplex. Amplicons were subsequently analysed on an ABI-3100 sequencer and allele sizes scored using the size standards Rox-350 or Rox-500 (GeneMapper 4.0; AppliedBiosystems, Inc.). We checked for genotyping errors due to stuttering, allelic dropout and null alleles with Micro-ChecKer 2.2.3 (van Oosterhout et al. 2004) and performed corrections when necessary.

Phylogenetic analyses, molecular dating and Bayesian phylogeographic reconstruction

Maximum-likelihood phylogenetic reconstructions (PhyML 3.0, Guindon \& Gascuel 2003) were performed on a concatenated data set of the two mitochondrial markers (1547 bp) and separately on the rag-1 data set
(737 bp). In both cases, we used a HKY+G+I model (JModelTest 0.1.1, Posada 2008) and tested the robustness of topologies by 1000 bootstrap replicates. We estimated the divergence time between major haplogroups from our cyt $b$ data set in BEAST 1.6.2 (Drummond \& Rambaut 2007), using a strict molecular clock (ucld.stdev parameter $<1$ with a frequency histogram abutting 0 when testing with a relaxed clock, BEAST manual version July 2007) and a coalescent prior (appropriate for intraspecific radiations). To decide which one to use, we performed short runs (1 chain of 5 million iterations) with the different coalescent priors available in BEAST and choose the one with the highest likelihood (coalescent: exponential growth). We used a HKY+G+I model of sequence evolution (JMoDelTest), and the tree was calibrated to the splits of $H$. meridionalis, $H$. savignyi/H. felix arabica, $H$. arborea and $H$. orientalis $/ H$. molleri (respectively, approximately 10, 6.2, 6.1, and 3.7 Mya, based on previous work, Smith et al. 2005; Stöck et al. 2012; using sequences available on GENBANK, see Table S3, Supporting information), with normally distributed priors. We ran three independent chains of 30 million iterations each and used TRACER 1.5 (http:// tree.bio.ed.ac.uk/software/tracer/) to check for convergence and combine the results.

We reconstructed the phylogeographic history of $H$. arborea by a Bayesian phylogeographic analysis of our mtDNA data set using spatial continuous diffusion models (Lemey et al. 2010) in BEAST 1.7.5 (Drummond et al. 2012), following the methodology recommended by Suchard \& Lemey (2013). Four different models were ran (homogeneous Brownian diffusion across branches, branch-specific diffusion rates (relaxedrandom walks, RRWs): Gamma RRW, Cauchy RRW and Lognormal RRW) during 100 million iterations (sampling every 10000 ), with a strict molecular clock (see above) and the Bayesian skyline plot as a flexible demographic prior. To perform model selection, we computed marginal likelihoods with 100 power posteriors along the path between prior and posterior (each of 100000 iterations following 10000 of burn-in, sampling every 1000) and estimated log marginal likelihoods using path and stepping stone sampling (shown to outperform other estimators, Baele et al. 2012, 2013). For the best-fitting diffusion model, the maximum clade credibility (MCC) tree was computed and annotated using the BEAST module TreeAnnotator 1.7.5. We then used SPREAD 1.0.4 (available: http://www. phylogeography.org/SPREAD.html) to project the MCC phylogeny in a spatial framework and summarized the full posterior distribution of trees to calculate the $80 \%$ highest posterior density (HPD) of node locations. Final results were visualized in GoOgLE EARTH (http://earth.google.com/). 


\section{Analyses of genetic structure}

For sequence data (concatenated mtDNA, rag-1), we built statistical parsimony networks with TCS 1.21 (Clement et al. 2000) which connects haplotypes under a parsimony limit (set to $95 \%$ ). For both data sets, the main haplogroups were defined with the individualbased, spatially explicit BAPS model (version 6.0, Corander et al. 2008) for clustering DNA sequence data (Cheng et al. 2013). This model combines sample locations with likelihood of the genetic data and is particularly efficient with large data sets (Cheng et al. 2013). We performed analyses using default parameters and following the software manual (version 21.12.2012). Several runs were repeated to ensure convergence and consistency of the results.

For microsatellites, we conducted individual-based assignment with the Bayesian algorithm of STRUCTURE 2.3.3 (Pritchard et al. 2000), using an admixture ancestry model with sampling locations as prior, and correlated allele frequencies between populations (as recommended for subtle population structure). Ten replicates (each consisting of $10^{5}$ iterations following a burn-in of $10^{4}$ ) were performed for every number of clusters $(K)$ between 1 and 11, from which we computed the corresponding $\Delta K$ ad hoc statistics (Evanno et al. 2005) with Structure Harvester 0.6.92 (Earl \& VonHoldt 2012, http:/ / taylor0.biology.ucla.edu/struct_harvest). Replicates were combined with CLUMPP (full search algorithm, Jakobsson \& Rosenberg 2007) and graphs of assignment probabilities built using DisTRUCT 1.1 (Rosenberg 2004). We conducted hierarchical analyses, by rerunning STRUCTURE within each of the major clusters, including populations assigned with a probability of at least $80 \%$. In addition, population differentiation was inferred by a principal component analysis (PCA) on allelic frequencies with PCAGEN 1.2 (http://www2. unil.ch/popgen/softwares/pcagen.htm), which evaluates the significance of axes by permutations. To estimate the level of isolation by distance, we also performed Mantel tests of genetic $\left(F_{\mathrm{ST}}\right)$ versus geographic distances (ARLEQUiN 3.5, Excoffier et al. 2005) across populations (where $n \geq 6$ individuals) assigned to the main groups defined by STRUCTURE (with a probability of at least $80 \%$ ).

\section{Analyses of genetic diversity}

For mtDNA and rag-1, we computed (ARLEQUiN) the haplotype $(\mathrm{Hd})$ and nucleotide diversity $(\pi)$ within populations where $n \geq 4$ sequences. For microsatellites where no null allele was detected, we assessed allelic richness and heterozygosity for populations where $n \geq 6$ genotyped samples with FSTAT, which performs a rarefaction procedure to a common sample per locus (Goudet 1995).

\section{Demographic analyses}

The demographic fluctuations of the main identified haplogroups were inferred from our sequence data sets (cyt b, D-loop, rag-1) by three separate approaches. First, we performed Bayesian coalescent-based analyses to evaluate the historical demographic fluctuations within each of the main haplogroups using the Extended Bayesian Skyline Plot (EBSP, Heled \& Drummond 2008) implemented in BEAST 1.6.2. The EBSP can combine several sequence sets (in our case: cyt b, D-loop, rag-1) and fits different demographic scenarios by allowing changes in population size overtime. For all three markers, we used HKY $+\mathrm{G}+\mathrm{I}$ models (JModelTeST). The clock rate $(\mu)$ of the $D$-loop and rag-1 was estimated from cyt $b$, where $\mu$ was fixed to the value previously obtained from the molecular calibration. Other parameters were either left as default or optimized for the EBSP (following Heled 2010), and chains were run for 60 million iterations. We used TRACER to assess burn-in and effective sample sizes (ESS) of parameters.

Second, we performed analyses of mismatch distributions, by comparing observed pairwise number of differences to distributions simulated under models of demographic (Schneider \& Excoffier 1999) and range expansions (Excoffier 2004), as implemented in ArLEQUIN. These models estimate the parameters of population expansion using a generalized least-square approach and compute their confidence intervals by bootstrapping (10 000 replicates in our case). Tests of goodness-of-fit (sum of squared deviation and Harpending's raggedness index) were computed to measure departures between observed and simulated distributions (ARLEQUIN). For each mitochondrial haplogroup, we estimated the time since expansion from the parameter $\tau(\tau=2 \mu \mathrm{t}$, where $\mu$ is the mutation rate, and $\mathrm{t}$ the time since expansion) and given the clock rates previously estimated by BEAST.

Finally, for mtDNA (concatenated cyt $b / D$-loop) and rag-1, we computed (DNASP 5) the following tests of selective neutrality within each of the main haplogroups: Fu's Fs, Tajima's D and Ramos-Onsins and Rozas's $R^{2}$, which has more statistical power for small sample sizes (Ramos-Onsins \& Rozas 2002). Their significances were tested by coalescent analyses (10 000 replicates).

\section{Intergroups gene flow}

To measure the level of gene flow between the main clusters, we calculated the demographic parameters $\theta$ 
( $\theta=x \mathrm{~N}_{\mathrm{e}} \mu$, where $\mathrm{N}_{\mathrm{e}}$ is the effective size, $\mu$ the mutation rate and $x$ a multiplier depending on the ploidy and the inheritance of the data: $x=1,2$ or 4 for mtDNA, haploid or diploid data, respectively) and $\mathrm{M}(\mathrm{M}=\mathrm{m} / \mu$, with $\mathrm{m}$ the immigration rate) from sequences (combining cyt $b, D$-loop, rag-1), and microsatellite data sets. From the product of $\theta$ and $M$, we can calculate the number of immigrant genes per generation $\left(x \mathrm{~N}_{\mathrm{e}} \mathrm{m}\right)$ from one cluster to another. Population boundaries can be defined when $x \mathrm{~N}_{\mathrm{e}} \mathrm{m}<1$ but values up to 25 can still reflect departure from panmixia (Waples \& Gaggiotti 2006). We delimited our candidate groups according to the main distribution of haplogroups and microsatellite clusters, and estimated $\theta$ and $M$ with Migrate 3.5.1 (full migration rates matrix, Beerli \& Felsenstein 2001) using the Bayesian inference search strategy (Beerli 2006). We first performed preliminary runs to optimize the prior distributions and burn-in periods. Relative mutation rates among loci were directly inferred from the data (through measures of Watterson's $\theta$; microsatellites) or calculated from the BEAST estimates (sequences). For microsatellites, we used the Brownian motion approximation to the ladder model, which gives very similar results to the stepwise-mutation model but with much faster parameter estimation (MigRATE manual, version 16.05.2012). In the final analyses, we ran one MCMC chain per locus (50 000 recorded genealogies among 5000000 visited), with burn-ins of 500000 (combined sequence data set) or 20000 steps (microsatellite data set). We monitored the effective sample sizes (ESS) of each parameter (including the likelihood) to make sure that the chains ran long enough (ESS > 1000, Migrate manual). Each data set was re-analysed several times to ensure the consistency of the results.

\section{Results}

Phylogenetic analyses, molecular dating and Bayesian phylogeographic reconstruction

Phylogenetic analyses of mtDNA (112 haplotypes, $1547 \mathrm{bp}$ ) revealed a supported clade restricted to the north-eastern Adriatic coast (H1-H7), and a paraphyletic group distributed across the rest of the range (Fig. S2, Supporting information). The rag-1 phylogeny (27 haplotypes) displayed a large polytomy involving our $H$. arborea sequences and several close relatives (H. molleri, $H$. intermedia and $H$. orientalis) with incomplete lineage sorting (Fig. S3, Supporting information). From cyt $b$ (estimated clock rate $=0.036$ substitution/ $\mathrm{My})$, we dated the divergence of the mitochondrial Adriatic isolate to be approximately 180 kya (95\% $\mathrm{CI}=70-300$; approximately two ice-ages). The time to the most recent common ancestor of the main clade roughly corresponds to the end of the last interglacial (90 kya, 95\% CI = 40-160). All chains yielded nearly identical estimates, suggesting convergence. Because of its unresolved phylogeny, we did not date divergence times between rag-1 haplogroups.

The pattern and timing of dispersal of $H$. arborea mitochondrial haplogroups were best inferred from the Lognormal RRW diffusion model (Table S5, Supporting information). The analysis estimated the location of ancestor sequences on the Adriatic coast, from which the southern Balkans were early colonized and then diversified, particularly south-east and north-west of the Pindus mountain range (Data S2). From the latter, Central and Western Europe were recently invaded by a first wave of colonization and frogs later expanded to northern areas. At the same time, the Hellenic peninsula and then Crete were fully colonized from central Greece. The analysis also depicted recent southward migrations, from Central Europe to the Balkans.

\section{Genetic structure and diversity}

The spatial clustering method of BAPS defined three major groups from our mtDNA data set (optimal partition, $\log ($ likelihood $)=-2622.5 ;$ Fig. 1): the Adriatic isolate (H1-7, haplogroup 1), sequences from southern and eastern Greece (H79-H112, haplogroup 2), and a widespread haplogroup distributed throughout the range (H8-78, haplogroup 3), with some geographic association of haplotypes (H31-45: only North and Western Europe; H54-59: only north-eastern Adriatic coast; H60-H78: only southern Balkans). Haplotype diversity (Hd) was greatly variable throughout the range (Table S2, Supporting information), but nucleotide diversity $(\pi)$ shows some geographic differences, being higher in the southern and western Balkans (Fig. 2b). Accordingly, the architecture of our mtDNA network (Fig. 1) was more complex for haplotypes occurring in the southern Balkans and on Crete (H60-H78, H79-H93), contrasting with the starlike shapes of haplotypes sampled further north (e.g. H94-H112, H54-H59, H8-H30, H31-H46).

Clustering analyses (BAPS) of the nuclear rag-1 (optimal partition: 4 groups, $\log ($ likelihood $)=-731.1$; Fig. 3 ) also distinguished western Balkans (H1-3, haplogroup 1) from southern Balkans (H4-H10, H13-H17, haplogroup 2) and the rest of the range (H18-27, haplogroup 3). A fourth cluster includes two haplotypes (H11-12, haplogroup 4) occurring in the most eastern populations from Romania (loc. 41), Serbia (loc. 21-22) and Greece (loc. 11), at the contact zone with $H$. orientalis and clustering with alleles from this latter species. Because this haplogroup probably originated from introgression events (no similar 


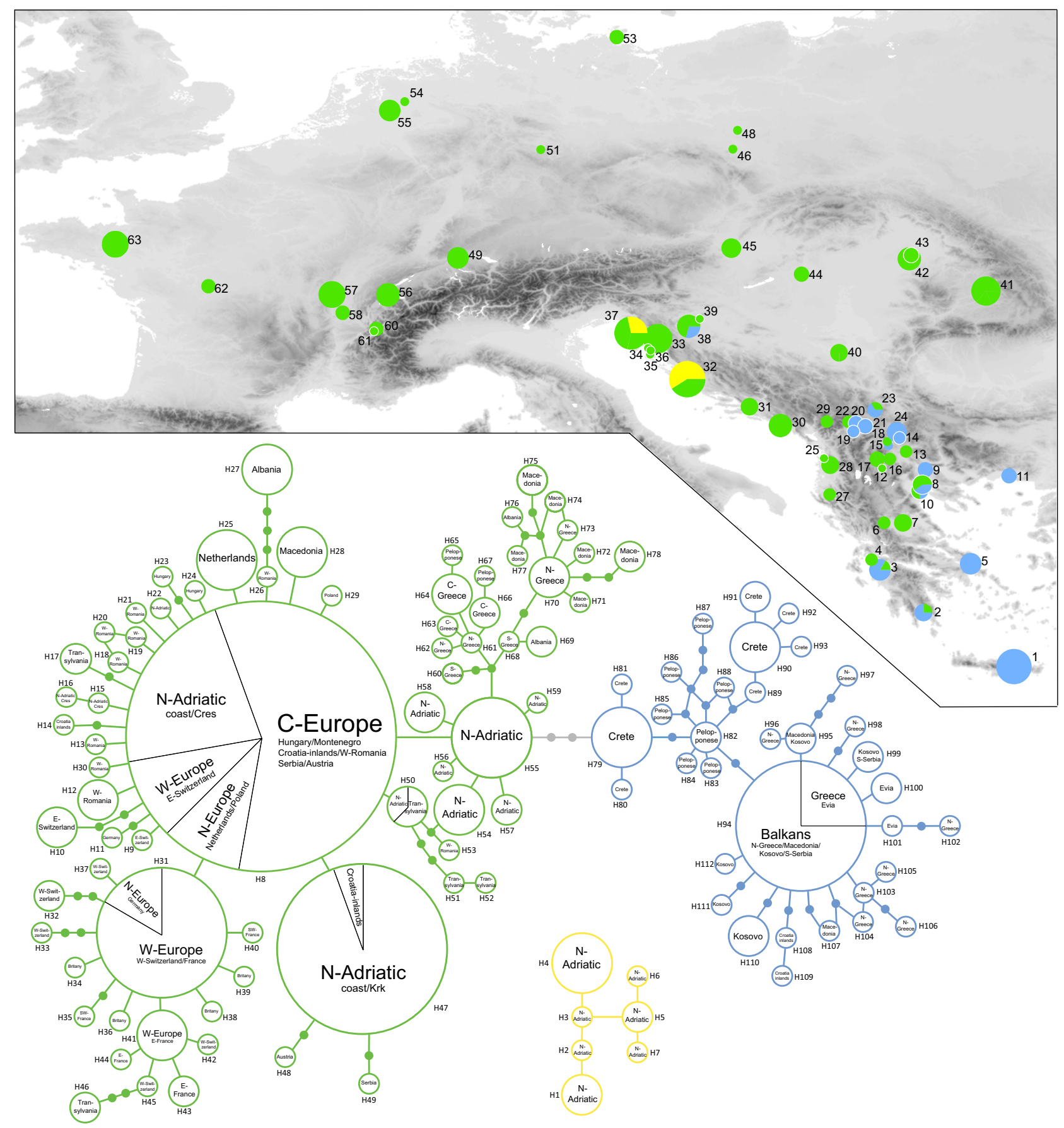

Fig. 1 Parsimony-based haplotype network of mitochondrial sequences (concatenated cyt $b+D$-loop) and distribution of the haplogroups defined by BAPS (yellow: haplogroup 1, blue: haplogroup 2, green: haplogroup 3). The following abbreviations are used: N: north; S: south; E: east; W: west; C: central. 'Macedonia' corresponds to the Former Yugoslav Republic of Macedonia.

cluster was found from the other markers), and also based on a very limited sample size, it was not included in the demographic analyses. The distribution of the rag1 diversity is similar as for mtDNA, where the most southern haplogroup (haplogroup 2) is also the richest (Table S2, Supporting information, Fig. 2c) and the most complex (Fig. 3). In both BAPS analyses, permuting sequences between groups resulted in substantial decreases in $\log$ (likelihood) (on average -42.6 for mtDNA, ranging from -9.4 to -79.1 ; on average -21.4 for rag-1, ranging from -1.4 to -42.6 ), suggesting robust assignments.

Individual-based clustering of microsatellite genotypes with StRUCTURE suggested two major groups 

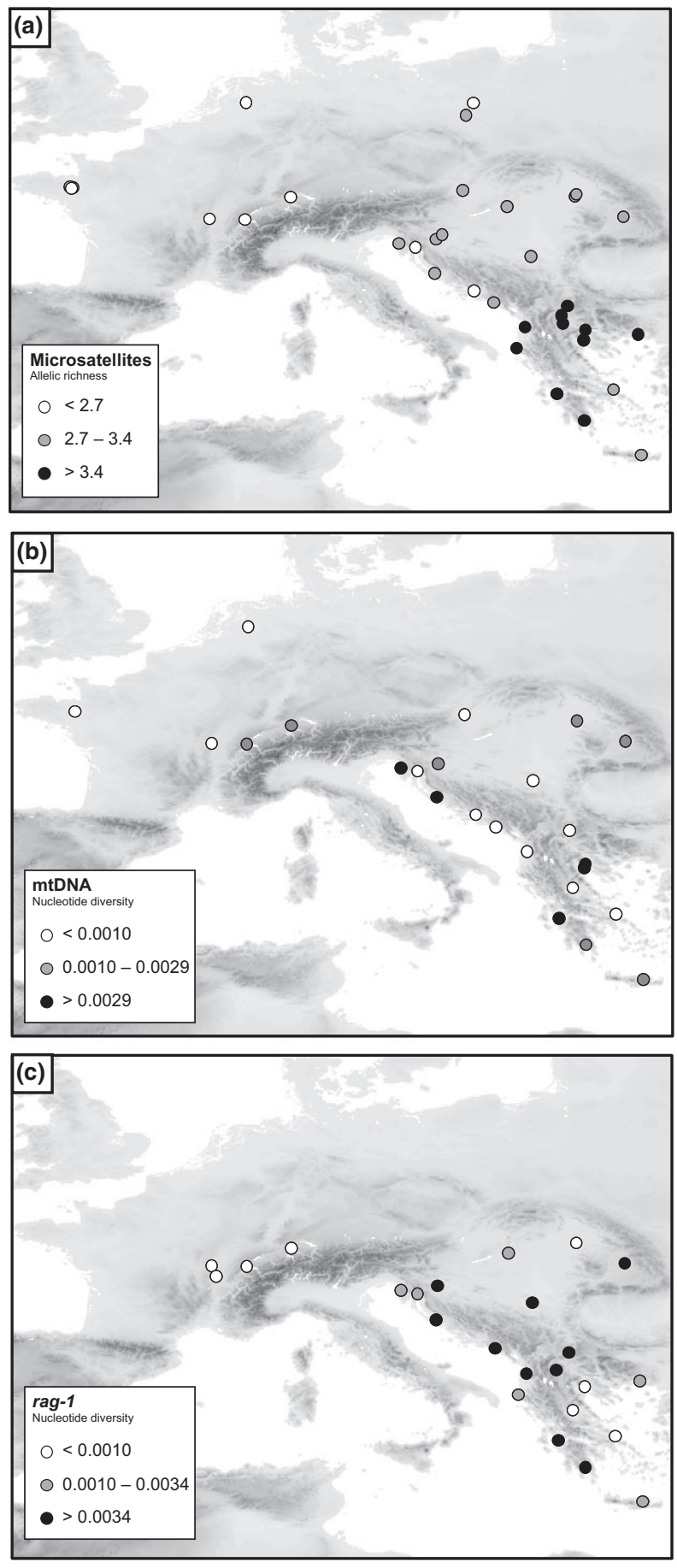

Fig. 2 Distribution of microsatellite allelic richness (scaled to 5 individuals; a) and mitochondrial (concatenated cyt $b+D$-loop; b) and rag-1 nucleotide diversity (c). Classes were built from natural breaks (Jenks) in ARcGIS 9.3 (ESRI). Details are available in Table S2 (Supporting information).

$(K=2$, highest $\Delta K=704.4 ; \quad$ Fig. $\quad$ S4, Supporting information) contrasting southern (loc. 1-7) and central/north-western (loc. 34-65) populations (Fig. 4).
Populations parapatric to these two groups featured intermediate probabilities of assignment (loc. 8-33). We could detect fine substructure within each cluster (Fig. 4). In southern Greece $(K=2$, highest $\Delta K=601.2$; Fig. S4, Supporting information), StRUCtURE distinguished Crete (loc.1) from Thessaly and Evia Island (loc. 4-7) with admixed populations in the Peloponnese Peninsula (loc. 2-3). In the rest of the range $(K=2$, highest $\Delta K=755.1$; Fig. S4, Supporting information), populations from France and western Switzerland (loc. 56-65) were differentiated from Central Europe (loc. 37-45). The populational PCA (Fig. S5, Supporting information) also contrasted Southern from Central Europe (N-S gradient, first axis) and depicted differentiation between eastern and western populations from the continental part of the range (E-W gradient, second axis). Both axes were significant. As null alleles were detected for several markers, especially in southern populations, we reran our analyses by (i) discarding the corresponding loci and (ii) considering null alleles as missing data. In both cases, results from STRUCTURE and the PCA remained unchanged. Finally, we found striking differences in microsatellite variability (allelic richness and heterozygosity) between the Balkans, Central and North-Western Europe, which are strongly decreasing with distances from southern areas (Table S2, Supporting information, Fig. 2a). Southern insular populations (Crete: loc.1, Evia: loc. 5) were also genetically poorer than neighbouring mainland areas (Table S2, Supporting information, Fig. 2a).

The correlation between pairwise $F_{\mathrm{ST}}$ values and geographic distances was significant for the central/northwestern microsatellite cluster (loc. 34-65; correlation coefficient $=0.74$; regression coefficient $=0.000158$ ), but not for the southern group (loc. 1-7).

\section{Demographic analyses}

The extended Bayesian skyline plot reconstructed a recent increase in population size for haplogroups 2 and 3 since the last glacial maximum (Fig. 5). Interestingly, the expansion was much stronger (100-fold increase) for haplogroup 3 than haplogroup 2 (10-fold increase) and indicated that the latter maintained a higher effective size during the last glaciation. In contrast, the $95 \%$ posterior distribution of the number of changes in population size (demographic.populationSizeChanges) does include 0 for haplogroup 1 (which is not the case for haplogroups 2 and 3). The unimodal distributions of pairwise nucleotide differences within the three main mtDNA and rag-1 haplogroups suggest past population expansions (Fig. 5). Based on the sum of squared differences and raggedness index, the fits to the spatial expansion model could never be rejected for 


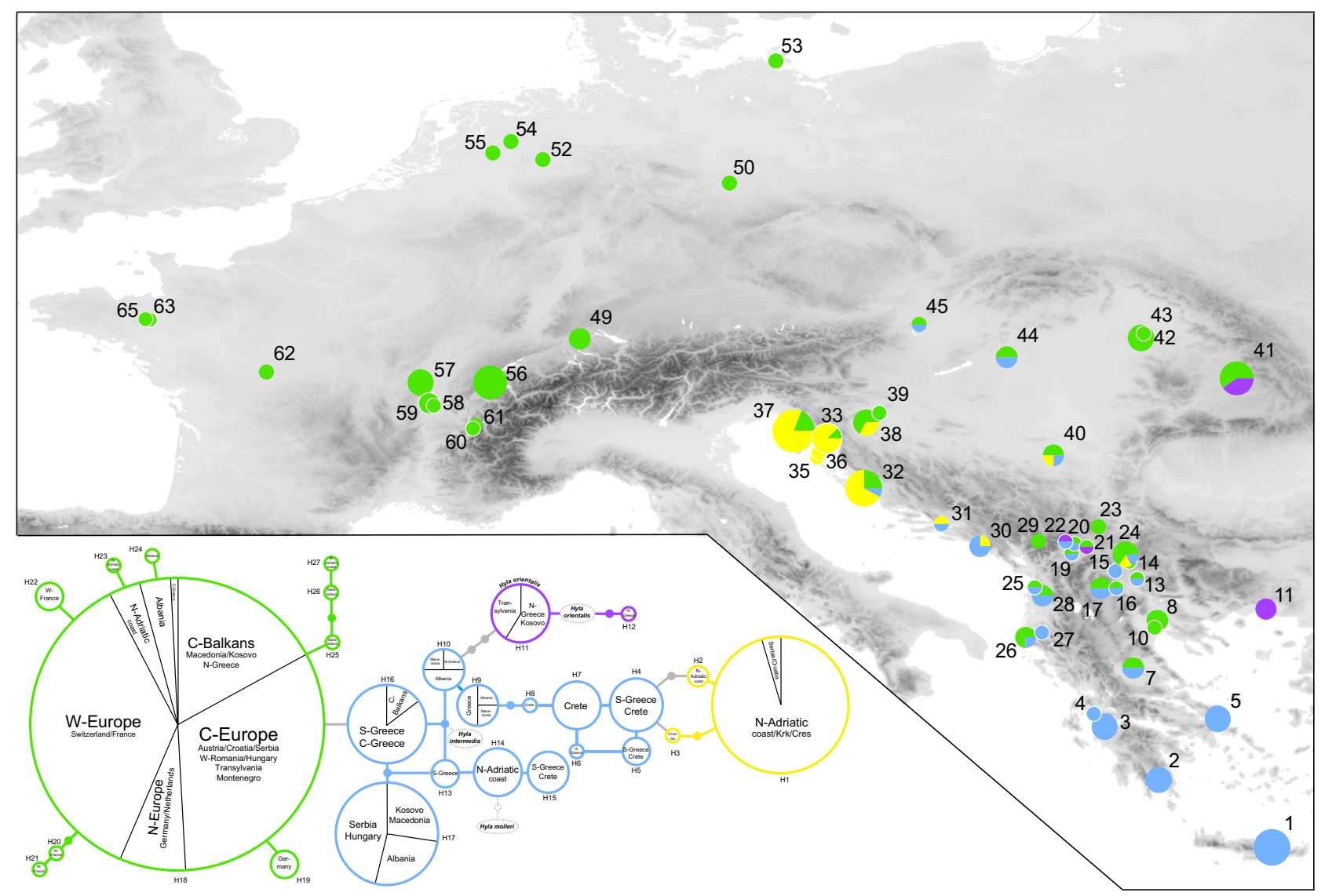

Fig. 3 Parsimony-based haplotype network of rag-1 nuclear sequences and distribution of the haplogroups defined by BAPS (yellow: haplogroup 1, blue: haplogroup 2, green: haplogroup 3, purple: haplogroup 4). Haplotypes sampled in closely related species are displayed. Abbreviations are the same as in Fig. 1.

any haplogroups of the two markers (Table 1). The mismatch distribution simulated under the model of demographic expansion was significantly different from observed distributions only for the rag-1 southern haplogroup 2 (Table 1). Calculated from the parameter $\tau$, the time since expansion pointed to the late-Pleistocene for all mitochondrial haplogroups (Table 1). Accordingly, tests for departure from neutrality were significant for the main mitochondrial haplogroups 2 and 3 but not for haplogroup 1 (Table 1). For rag-1, departure from neutral values could only be ascertained for haplogroup 3 (Table 1).

\section{Intergroups gene flow}

Because the distributions of nuclear and mitochondrial haplogroups/clusters defined by BAPS and STRUCTURE were congruent, but not strictly identical across markers (with many admixed localities), it was difficult to define precise spatial boundaries between the three main genetic groups. Therefore, we delimited candidate populations according to the geographic regions where these haplogroups principally occurred: southern Balkans (loc. 1-31), northern Adriatic (loc. 32-37) and Central/North-Western Europe (loc. 38-65). Multiple runs of MigRATE performed on this framework led to similar estimates, and all parameters had unimodal posterior distributions, suggesting that single optima were reached. Because the posterior distribution abutted 0 for most parameters, we preferred the modes to the medians for calculating the demographic estimator $x \mathrm{~N}_{\mathrm{e}} \mathrm{m}(=\theta \times \mathrm{M})$. Migration estimates $\left(x \mathrm{~N}_{\mathrm{e}} \mathrm{m}\right)$ ranged from 0.6 to 8.5 for sequence data and from 6.2 to 19.2 for microsatellites (Fig. S6, Supporting information), which is below the cut-off values for departures from panmixia $\left(x \mathrm{~N}_{\mathrm{e}} \mathrm{m}<25\right)$. The estimated gene flows were mostly asymmetric, with the expanding groups (southern Balkans, Central/North-Western Europe) providing migrants to the Adriatic populations (Fig. S6, Supporting information). Table S6 (Supporting information) displays the parameters estimated by Migrate and their 95\% posterior distribution. 


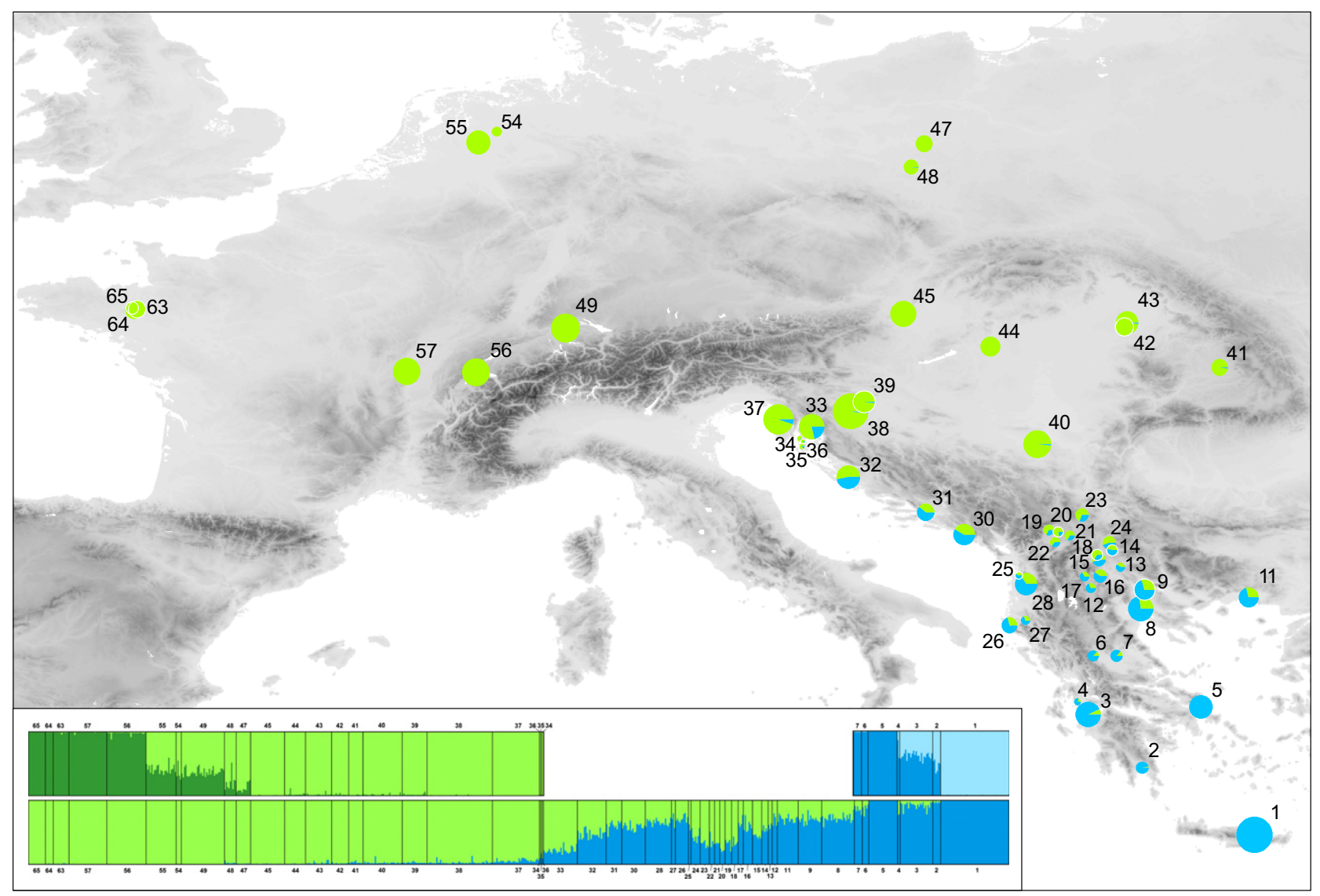

Fig. 4 Genotyped-based assignments of Hyla arborea individuals, based on Bayesian clustering analyses of 30 microsatellites (STRUCTURE). Barplots represents the assignment probability of each individual. The lower chart includes all samples $(K=2$, see main text and Fig. S4, Supporting information). The upper charts correspond to hierarchical analyses on each of the two main clusters (upper left: Central/North-Western Europe, $K=2$; upper right: southern Greece, $K=2$ ). The map shows the mean assignment probability of every locality to each of the main clusters.

\section{Discussion}

Our study provides a fine-scale reconstruction of the phylogeography of the widespread European tree frog H. arborea. Our dense sampling and multilocus approach allowed us to uncover hidden diversity, and were especially suitable to test assumptions regarding the distribution of genetic variation and its implications for conservation. First, cryptic structure could be documented across the Balkan Peninsula, allowing us to check whether the recently diverged groups match the commonly used criteria for ESUs and evolutionary populations. Second, we could accurately assess the geographic patterns of diversity and map them to the regional levels of vulnerability.

\section{Refugia within refugia: insights into the Balkans biogeography}

One aim of our study was to test whether southern areas exhibit cryptic structuring, as previously suspected (Stöck et al. 2008b, 2012) and expected under the 'refugia within refugia' paradigm (Gómez \& Lunt 2007). Indeed, although there were a few discrepancies on the precise boundaries, our mitochondrial and nuclear data sets were largely congruent in support of a late-Pleistocene diversification. This involved several major genetic groups in southern areas (western Adriatic coast; southern Balkans), of which one recently expanded across the rest of the range, from the Balkans to Western Europe. The only exception came from the Adriatic group, which does not significantly stand out from our microsatellite data set, maybe because of recent backcrossing by southern and Central European immigrants (see below). In addition, it is unclear whether the fourth rag-1 haplogroup found in the most eastern populations stem from an ancestral polymorphism or (more probably) from introgressive hybridization with the proximate H. orientalis (Stöck et al. 2012), which displays closely related haplotypes (Fig. 3). We further detected subtle structure from our microsatellite data set (consistent with spatial association of mtDNA 

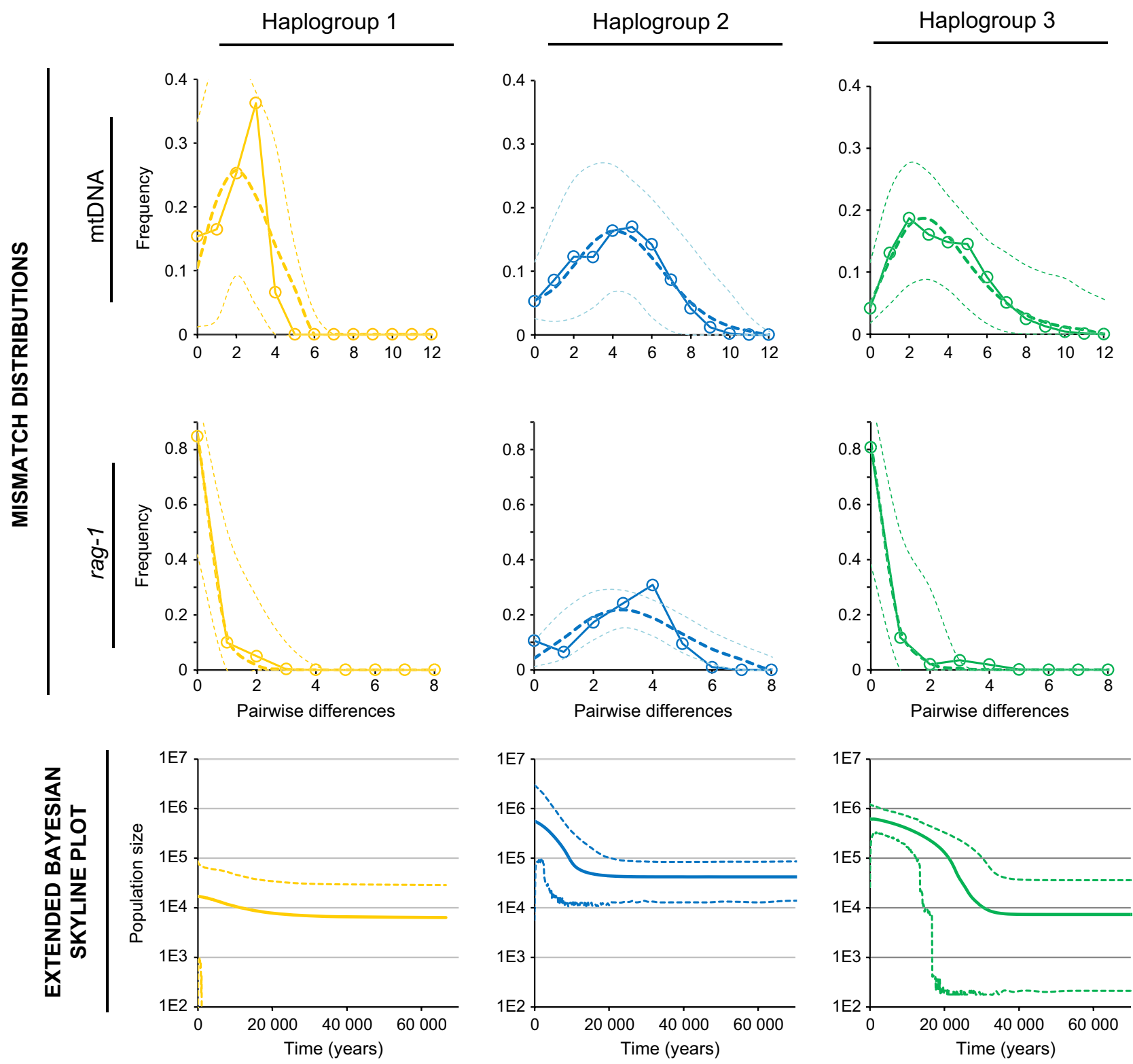

Fig. 5 Demographic analyses of the three main haplogroups defined from sequence data. Upper charts display the distribution of observed (circles) and expected (bold dash lines) pairwise nucleotide differences under models of sudden demographic expansion (ARLEQuin 3.5). 95\% Confidence intervals of the expected distributions are indicated (thin dash lines). Mismatch distributions simulated under models of spatial expansion yielded similar expected curves (not shown). Tests for goodness-of-fit are available in Table 1. Lower charts display extended Bayesian skyline plots (EBSP) representing the recent demographic trends of each haplogroups, based on combined sequence data (solid lines: median estimates, dash lines: $95 \%$ confidence intervals). Y axes display estimated population size, as the product of effective population size per generation length.

haplotypes), pointing out recent contact between Crete and the Peloponnese (probably when sea levels dropped in the Ionian Sea; Perissoratis \& Conispoliatis 2003), and population differentiation across recently colonized areas (Central, North-Western Europe) which might stem from isolation by distance (as reported), gene surfing (Excoffier et al. 2009) or post-glacial climate and range changes (e.g. the Younger Dryas, 11 kya; see
Taberlet \& Cheddadi 2002 and references therein). The latter could particularly explain why the most northern areas were colonized by a last, most recent wave of invasion, as depicted by the mtDNA phylogeographic inference.

Our findings are thus in line with the view that southern refugial areas host 'refugia within refugia' (Gómez \& Lunt 2007), generating cryptic structure and maintaining 


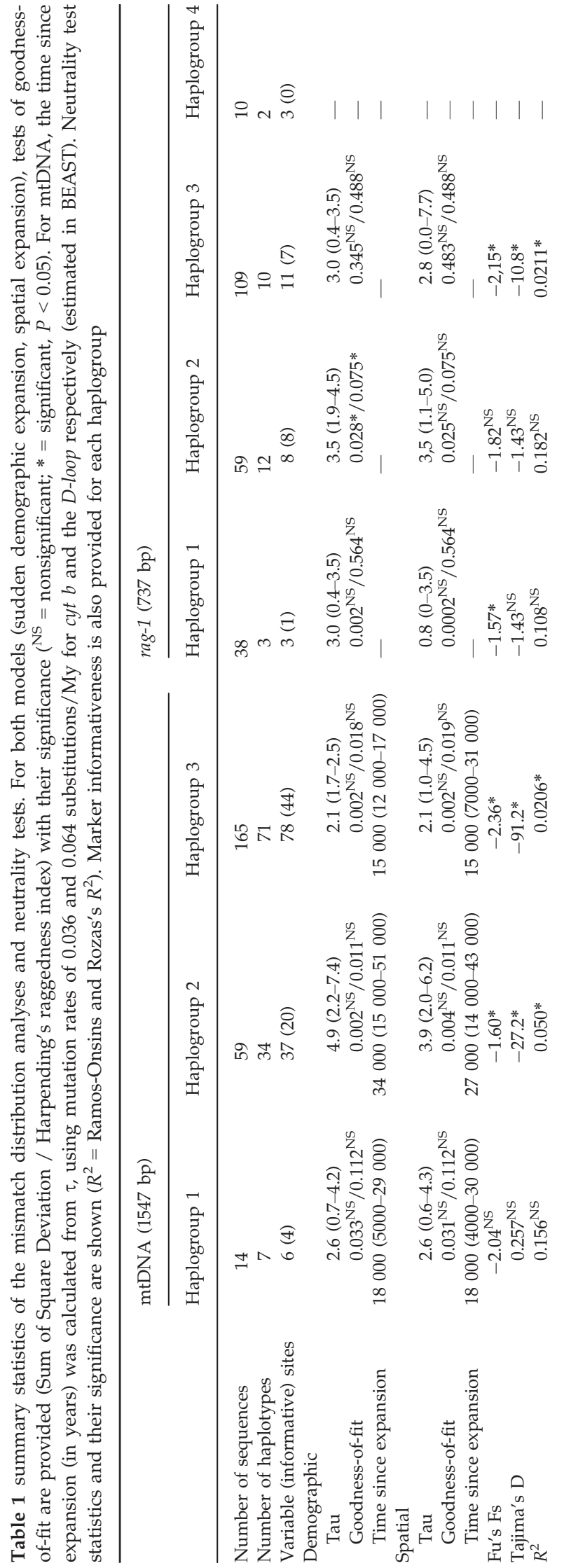

high genetic diversity. Increasing empirical evidence in support of this pattern is coming from taxa across a diversity of regions (Iberia: e.g. Martínez-Solano et al. 2006; Rowe et al. 2006; Gonçalves et al. 2009; Italy: e.g. Canestrelli et al. 2012; Nearctic: e.g. Nielson et al. 2001). So far, the Balkans have received less attention (Hewitt 2011), but signatures of multiple refugia have been found for a few species, both in Mediterranean (e.g. Ursenbacher et al. 2008; Bužan et al. 2010) and nonMediterranean parts of the Peninsula (Provan \& Bennett 2008; e.g. Kryštufek et al. 2009; Fijarczyk et al. 2011). In particular, the northern Adriatic coast seems to have acted as a remote sanctuary for several Balkanic species (e.g. green toads, Stöck et al. 2008a; nose-horned viper, Ursenbacher et al. 2008), including tree frogs. There and in other parts (i.e. southern Balkans), population maintenance might have been tightly linked to the glacial distribution of deciduous forests (supposedly restricted to the southern and eastern Balkans, the Carpathian belt and south of the Alpine Glaciers, Adams \& Faure 1997), and post-glacial expansions were probably associated with the recolonization of tree species (Taberlet \& Cheddadi 2002; Stöck et al. 2012). Our results then support the view that the Balkan Peninsula is an important centre of European biodiversity, especially for the herpetofauna (Crnobrnja-Isailović 2007).

Multiple subrefugia are typical for low-vagility organisms such as newts (Sotiropoulos et al. 2007) or snakes (Ursenbacher et al. 2008) and are usually easier to detect given that populations have experienced long periods of allopatry. In contrast, tree frogs have a relatively high dispersal capacity for an amphibian (up to $4 \mathrm{~km} /$ year, Vos et al. 2000) so that ancestral populations may have regularly merged during interglacials, engulfing traces of multiple refugia (e.g. Stöck et al. 2012). The increasing appeal to multilocus genomic data will give exciting insights into the glacial and post-glacial phylogeography of genetically uniform taxa that might similarly hide cryptic population differentiations (Emerson et al. 2010).

\section{Defining recently diverged management units}

An important question raised by this cryptic diversity is whether conservation units can be defined from such recently diverged lineages, that is, do they match the criteria for ESUs or evolutionary populations' paradigms? Evolutionarily significant units can be defined provided that genetic distinctiveness of populations matches other features (e.g. geographic distribution, adaptive phenotypic variation) and is supported by mitochondrial monophyly and reciprocal significant nuclear divergence (Moritz 1994). Here, we did find some concordance between multiple molecular 
polymorphisms and their geographic distribution. However, only the Adriatic mitochondrial clade was significantly supported, and populations carrying this mitochondrial lineage did not form a distinct cluster based on microsatellites. Our groups also exhibited considerable mixing where they come into contact, consistent with ongoing gene flow. Therefore, these clusters hardly fulfil the requirements of ESUs. On the other hand, despite a rough choice of population boundaries that should have led to overestimate the amount of gene flow (particularly since haplogroup 2 and 3 seem to have originated from proximate areas), patterns still reflect a departure from panmixia: even though most migration estimates are above the stringent cut-off value of $x \mathrm{~N}_{\mathrm{e}} \mathrm{m}<1$, they indicate isolation with migration $\left(x \mathrm{~N}_{\mathrm{e}} \mathrm{m}<25\right)$ compatible with the evolutionary population concept proposed by Waples \& Gaggiotti (2006).

Thus, although our clusters might be too young and insufficiently resolved to be considered ESUs, they feature enough genetic isolation to deserve special attention for wildlife management. The demographic approach thus seems more powerful to assess the evolutionary significance and conservation values of young subspecific radiations. Nevertheless, management design based only on the levels of gene flow may still miss significant divergences. In our case, the northern Adriatic coast can be viewed as a hotspot of genetic diversity, but experienced the highest levels of migration. New estimators combining both phylogeographic and demographic information (as well as adaptive variation, when applicable) might allow considering all valuable entities. In addition, the future development of specific criteria for discriminating different levels of population cohesion would be useful to set priorities for the definition of conservation units (Reilly et al. 2012).

\section{Biogeographic loss of diversity and regional vulnerability}

One main goal of our study was to assess whether the vulnerability of Western European populations was consistent with a biogeographic loss of genetic diversity. Higher amounts of microsatellite and sequence polymorphisms were indeed found in Southern Europe, which, from our analyses, seems largely explained by the maintenance of distinct ancestral demes and demographic stability of these populations. We could also document post-glacial expansions across most parts of the range (see also Stöck et al. 2012), leading to a severe loss of variability resulting from colonization-associated drift (Excoffier et al. 2009). Thus, the low levels of genetic diversity in Western and Northern Europe, also reported from previous regional studies (Edenhamn et al. 2000; Andersen et al. 2004; Arens et al. 2006; Dubey et al. 2009), might stem from historic/phylogeographic reasons more than from human disturbances.

From our results, this geographic trend in genetic diversity approximately corresponds to regional conservation status: south-eastern $H$. arborea populations are not considered threatened, which clearly contrasts with the precarious situation of the species in the north and west of Europe (Fig. S1, Supporting information). Although the level of threat must largely depend on the intensity of disturbances (especially land use and pollution), it is tempting to suggest that biogeographic history predisposes population vulnerability to current environmental pressures, even though this cannot be formally tested with the data in hand. Such natural genetic impoverishment might impact the sensitivity of populations to identified threats, like habitat destruction and pesticide exposure (an alarming cause of decline, Brühl et al. 2013), and accelerate their deterioration. Furthermore, Northern and Western European amphibian communities are especially challenged by the chytrid fungus, which does not yet affect Balkanic regions (RACE 2013). Whether the amount of genetic diversity conditions the susceptibility to infection is unclear (May et al. 2011), but it is known to improve fitness in our study species (Luquet et al. 2011). Experimental work aiming to understand the interaction between variability, fitness and known threats (e.g. pollutants) would be particularly relevant to test these hypotheses (e.g. Pearman \& Garner 2005).

A few studies have empirically explored the link between genetic variation and population trends in plants (e.g. Brütting et al. 2012) and animals (e.g. Kvist et al. 2011). However, to our knowledge, only Schmitt \& Hewitt (2004) had previously investigated this relationship in a biogeographic context. These authors showed that European butterflies performed better in refugial areas (eastern and southern Europe) than in recently deglaciated regions and discussed the lack of adaptability of the genetically poor northern and western populations. Many widespread species thrive in the central part of their distribution but are regionally endangered at the periphery (Hoffmann \& Blows 1994). This range limit effect might have biogeographic origins in postglacial areas, at least in species where differences in genetic variation could be detected across the range (Channell 2004).

Future studies should not only assess the role of landscape changes, but also account for the basal diversity historically present in a region. Especially, largescale phylogeographic surveys of widespread taxa, where knowledge of regional population dynamics is available, would be most relevant to address this issue. In our case, we benefited from the relatively 
well-covered conservation situation of $H$. arborea (although assessment data were too heteroclite for accurate analyses), most likely because it can be considered as an umbrella species, and populations are easy to detect and monitor by call census (Pellet \& Schmidt 2005). Amphibians are especially good candidates to test this relationship, because they seem particularly susceptible to diversity loss (Allentoft \& O'Brien 2010).

Phylogeographic analyses such as ours thus lay the ground work for conservation planning and identification of the potentially most sensitive regions. Even without fine-scale genetic data, general ideas of the main historical events (e.g. range expansion from a putative refugia) could be used to draw assumptions regarding their impact on diversity. In complement to the commonly used criteria (observed and forecast species abundance, population trends, potential threats, e.g. IUCN 2001), we propose that the geographic distribution (i.e. refugial vs post-glacial) should be considered when assessing regional risks and red lists.

\section{Acknowledgements}

We thank the numerous people who contributed to the sampling (credited in Table S3, Supporting information) and shared information on $H$. arborea conservation status (credited in Table S1, Supporting information). B. Pasteur and R. Sermier also provided substantial help with the laboratory work. We also thank the Nature Protection Directorate of the Croatian Ministry of Culture, the Greek Ministry of Environment and the Serbian Ministry for Nature Protection for permission to collect DNA samples. We are grateful to S. Dubey, along with three anonymous reviewers for useful suggestions on previous versions of the manuscript. This study was founded by the Swiss National Science Foundation (grant 31003A_129894 to $\mathrm{NP}$ ) and the University of Lausanne (PhD fellowship from the Faculty of Biology and Medicine, grants from the Fondation Agassiz and the Fondation du $450^{\mathrm{e}}$ to CD). JCI was supported by grant No 173025 Ministry of Education and Science of Republic of Serbia.

\section{References}

Adams JM, Faure H (1997) Review and Atlas of Palaeovegetation: Preliminary land ecosystem maps of the world since the Last Glacial Maximum. Oak Ridge National Laboratory, Oak Ridge, Tennessee. Available from: http://www.esd.ornl. gov/projects/qen/nerc.html

Allentoft ME, O'Brien J (2010) Global amphibian declines, loss of genetic diversity and fitness: a review. Diversity, 2, 47-71.

Andersen LW, Fog K, Damgaard C (2004) Habitat fragmentation causes bottlenecks and inbreeding in the European tree frog (Hyla arborea). Proceedings of the Royal Society B: Biological Sciences, 271, 1293-1302.

Arens P, Van't Westende W, Bugter R et al. (2000) Microsatellite markers for the European tree frog Hyla arborea. Molecular Ecology, 9, 1944-1946.
Arens P, Bugter R, Van't Westende W et al. (2006) Microsatellite variation and population structure of a recovering tree frog (Hyla arborea L.) metapopulation. Conservation Genetics, 7, 825-834.

Baele G, Lemey P, Bedford T et al. (2012) Improving the accuracy of demographic and molecular clock model comparison while accommodating phylogenetic uncertainty. Molecular Biology and Evolution, 29, 2157-2167.

Baele G, Li WLS, Drummond AJ et al. (2013) Accurate model selection of relaxed molecular clocks in Bayesian phylogenetics. Molecular Biology and Evolution, 30, 219-243.

Beebee TJC (2005) Conservation genetics of amphibians. Heredity, 95, 423-427.

Beerli P (2006) Comparison of Bayesian and maximum likelihood inference of population genetic parameters. Bioinformatics, 22, 341-345.

Beerli P, Felsenstein J (2001) Maximum likelihood estimation of a migration matrix and effective population sizes in $\mathrm{n}$ subpopulations by using a coalescent approach. Proceedings of the National Academy of Sciences, 98, 4563-4568.

Berset-Brändli L, Jaquiéry J, Broquet T et al. (2008a) Isolation and characterization of microsatellite loci for the European tree frog (Hyla arborea). Molecular Ecology Resources, 8, 1095-1097.

Berset-Brändli L, Jaquiéry J, Broquet T et al. (2008b) Extreme heterochiasmy and nascent sex chromosomes in European tree frogs. Proceedings of the Royal Society B: Biological Sciences, 275, 1577-1585.

Brelsford A, Stöck M, Betto-Colliard C et al. (2013) Homologous sex chromosomes in three deeply divergent anuran species. Evolution, 67, 2434-2440.

Broquet T, Berset-Brändli L, Emaresi G et al. (2007) Buccal swabs allow efficient and reliable microsatellite genotyping in amphibians. Conservation Genetics, 61, 1219-1228.

Brühl CA, Schmidt T, Pieper S et al. (2013) Terrestrial pesticide exposure of amphibians: an underestimated cause of global decline? Scientific Reports, 2, 1135.

Brütting C, Wesche K, Meyer S (2012) Genetic diversity of six arable plants in relation to their Red List status. Biodiversity and Conservation, 21, 745-761.

Buza L, Young A, Thrall P (2000) Genetic erosion, inbreeding and reduced fitness in fragmented populations of the endangered tetraploid pea Swainsona recta. Biological Conservation, 93, 177-186.

Bužan EV, Förster DW, Searle JB et al. (2010) A new cytochrome $b$ phylogroup of the common vole (Microtus arvalis) endemic to the Balkans and its implications for the evolutionary history of the species. Biological Journal of the Linnean Society, 100, 788-796.

Canestrelli D, Salvi D, Maura M et al. (2012) One species, three Pleistocene evolutionary histories: phylogeography of the Italian Crested Newt, Triturus carnifex. PLoS ONE, 7, e41754.

Channell R (2004) The conservation value of peripheral populations: the supporting science. In: Proceedings of the species at risk 2004 Pathways to recovery conference (ed. Hooper TD), March 2-6 2004, Victoria B.C. Species at risk 2004 Pathway to recovery conference organizing committee. Victoria B.C.

Cheng L, Connor TR, Sirén J et al. (2013) Hierarchical and spatially explicit clustering of DNA sequences with BAPS software. Molecular Biology and Evolution, 30, 1224-1228. 
Clement M, Posada D, Crandall KA (2000) TCS: a computer program to estimate gene genealogies. Molecular Ecology, 9, 1657-1660.

Corander J, Sirén J, Arjas E (2008) Bayesian Spatial modeling of genetic population structure. Computational Statistics, 23, 111129.

Crnobrnja-Isailović J (2007) Cross-section of a refugium: genetic diversity of amphibian and reptile populations in the Balkans. In: Phylogeography of Southern European Refugia (eds Weiss S, Ferrand N), pp. 327-337. Springer, Amsterdam, Netherlands.

van Dongen S, Backeljau T, Matthysen E et al. (1998) Genetic population structure of the winter moth (Operophtera brumata L.) (Lepidoptera, Geometridae) in a fragmented landscape. Heredity, 80, 92-100.

Drummond AJ, Rambaut A (2007) BEAST: Bayesian evolutionary analysis by sampling trees. BMC Evolutionary Biology, 7, 214

Drummond AJ, Suchard MA, Xie D, Rambaut A (2012) Bayesian phylogenetics with BEAUTi and the BEAST 1.7. Molecular Biology and Evolution, 29, 1969-1973.

Dubey S, Ursenbacher S, Pellet L et al. (2009) Genetic differentiation in two European tree frog (Hyla arborea) metapopulations in contrasted landscapes of western Switzerland. Amphibia-Reptilia, 30, 127-133.

Earl DA, VonHoldt BM (2012) STRUCTURE HARVESTER: a website and program for visualizing STRUCTURE output and implementing the Evanno method. Conservation Genetics Resources, 4, 359-361.

Edenhamn P, Höggren M, Carlson A (2000) Genetic diversity and fitness in peripheral and central populations of the European tree frog Hyla arborea. Hereditas, 133, 115-122.

Emerson K, Merz CR, Catchen JM et al. (2010) Resolving postglacial phylogeography using high-throughput sequencing. Proceedings of the National Academy of Sciences, 107, 16196-16200.

Epps CW, Paslbøll PJ, Wehausen JD et al. (2005) Highways block gene flow and causes a rapid decline in genetic diversity of desert bighorn sheep. Ecology Letters, 8, 1029-1038.

Evanno G, Regnaut S, Goudet J (2005) Detecting the number of clusters of individuals using the software STRUCTURE: a simulation study. Molecular Ecology, 14, 2611-2620.

Excoffier L (2004) Patterns of DNA sequence diversity and genetic structure after a range expansion: lessons from the infinite-island model. Molecular Ecology, 13, 853-864.

Excoffier L, Laval G, Schneider S (2005) ArLequin ver. 3.0: an integrated software package for population genetics data analysis. Evolutionary Bioinformatics Online, 1, 47-50.

Excoffier L, Foll M, Petit RJ (2009) Genetic consequences of range expansions. Annual Review of Ecology, Evolution and Systematics, 40, 481-501.

Fijarczyk A, Nadachowska K, Hofman S et al. (2011) Nuclear and mitochondrial phylogeography of the European firebellied toads Bombina bombina and Bombina variegata supports their independent histories. Molecular Ecology, 20, 3381-3398.

Frankham R (2005) Genetics and extinction. Biological Conservation, 125, 131-140.

Goebel AM, Donnelly J, Atz M (1999) PCR primers and amplification methods for the $12 \mathrm{~S}$ ribosomal DNA, cytochrome oxidase I, cytochrome $\mathrm{b}$, the control region in bufonids and other frogs and an overview of PCR primers available for analyses of amphibians. Molecular Phylogenetics and Evolution, 11, 163-199.

Gómez A, Lunt DH (2007) Refugia within refugia: patterns of phylogeographic concordance in the Iberian Peninsula. In: Phylogeography of Southern European Refugia (eds Weiss S, Ferrand N), pp. 155-188. Springer, Amsterdam, Netherlands.

Gonçalves H, Martínez-Solano I, Pereira RJ et al. (2009) High levels of population subdivision in a morphologically conserved Mediterranean toad (Alytes cisternasii) result from recent, multiple refugia: evidence from mtDNA, microsatellites and nuclear genealogies. Molecular Ecology, 18, 51435160.

Goudet J (1995) FSTAT (version 1.2): a computer program to calculate F-Statistics. Journal of Heredity, 86, 485-486.

Gouy M, Guindon S, Gascuel O (2010) SEAVIEW version 4: a multiplatform graphical user interface for sequence alignment and phylogenetic tree building. Molecular Biology and Evolution, 2, 221-224.

Guindon S, Gascuel O (2003) PHYML: a simple, fast and accurate algorithm to estimate large phylogenies by maximum likelihood. Systematic Biology, 52, 696-704.

Hansson B, Westerberg L (2002) On the correlation between heterozygosity and fitness in natural populations. Molecular Ecology, 11, 2467-2474.

Heled J (2010) Extended bayesian skyline plot tutorial. Available from: tutorial.east.bio.ed.ac.uk/Tutorials

Heled J, Drummond AJ (2008) Bayesian inference of population size history from multiple loci. BMC Evolutionary Biology, 8, 289.

Hewitt GM (2000) The genetic legacy of the quaternary ice ages. Nature, 405, 907-913.

Hewitt GM (2011) Mediterranean peninsulas: the evolution of hotspots. In: Biodiversity Hotspots (eds Zachos FE, Habel JC), pp. 123-147. Springer, Berlin, Germany.

Hoffmann AA, Blows MW (1994) Species borders: ecological and evolutionary perspectives. Trends in Ecology and Evolution, 9, 223-227.

Hughes AR, Inouye BD, Johnson MTJ et al. (2008) Ecological consequences of genetic diversity. Ecology Letters, 11, 609-623.

IUCN (2001) IUCN Red List Categories and Criteria, Version 3.1 2nd edn. IUCN Species Survival Commission. IUCN, Gland, Switzerland. Available from: http://www.iucnredlist.org/ technical-documents/categories-and-criteria

Jakobsson M, Rosenberg NA (2007) CLUMPP: a cluster matching and permutation program for dealing with label switching and multimodality in analysis of population structure. Bioinformatics, 23, 1801-1806.

Kryštufek B, Bryja J, Bužan EV (2009) Mitochondrial phylogeography of the European ground squirrel, Spemophilus citellus, yields evidence on refugia for steppic taxa in the southern Balkans. Heredity, 103, 129-135.

Kvist L, Giralt D, Valera F et al. (2011) Population decline is accompanied by loss of genetic diversity in the Lesser Grey Shrike Lanius minor. Ibis, 153, 98-109.

Lemey P, Rambaut A, Welch JJ, Suchard MA (2010) Phylogeography takes a relaxed random walk in continuous space and time. Molecular Biology and Evolution, 27, 1877-1885.

Librado P, Rozas J (2009) DNASP v5: a software for comprehensive analysis of DNA polymorphism data. Bioinformatics, 25 1451-1452. 
Luijten SH, Dierick A, Gerard J et al. (2000) Population size, genetic variation, and reproductive success in a rapidly declining, self-compatible perennial (Arnica montana) in the Netherlands. Conservation Biology, 14, 1776-1787.

Luquet E, David P, Léna JP et al. (2011) Heterozygosity-fitness correlations among wild populations of European tree frog (Hyla arborea) detect fixation load. Molecular Ecology, 20, 1877-1887.

Madsen T, Olsson M, Wittzell H et al. (2000) Population size and genetic diversity in sand lizards (Lacerta agilis) and adders (Vipera berus). Biological Conservation, 94, 257-262.

Martínez-Solano I, Teixeira J, Buckley D et al. (2006) Mitochondrial DNA phylogeography of Lissotriton boscai (Caudata, Salamandridae): evidence for old, multiple refugia in an Iberian endemic. Molecular Ecology, 15, 3375-3388.

May S, Zeisset I, Beebee TJC (2011) Larval fitness and immunogenetic diversity in chytrid-infected and uninfected natterjack toad (Bufo calamita) populations. Conservation Genetics, 12, 805-811.

Meagher S (1999) Genetic diversity and Capillaria hepatica (Nematoda) prevalence in Michigan dear mouse populations. Evolution, 53, 2033-2042.

Moritz C (1994) Defining 'Evolutionarily Significant Units' for conservation. Trends in Ecology and Evolution, 9, 373-375.

Newman D, Pilson D (1997) Increased probability of extinction due to decreased genetic effective population size: experimental populations of Clarkia pulchella. Evolution, 51, 354-362.

Nielson M, Lohman K, Sullivan J (2001) Phylogeography of the tailed frog (Ascaphus truei): implications for the biogeography of the Pacific Northwest. Evolution, 55, 147-160.

van Oosterhout C, Hutchinson WF, Wills DP et al. (2004) MiCRO-CHECKER: software for identifying and correcting genotyping errors in microsatellite data. Molecular Ecology Notes, 4, 535-538.

Oostermeijer JGB, van Eijck MW, van Leeuwen NC et al. (1995) Analysis of the relationship between allozyme heterozygosity and fitness in the rare Gentiana pneumonanthe L. Journal of Evolutionary Biology, 8, 739-759.

Pearman PB, Garner WJ (2005) Susceptibility of Italian agile frog populations to an emerging strain of Ranavirus parallels population genetic diversity. Ecology Letters, 8, 401-408.

Pellet J, Schmidt BR (2005) Monitoring distributions using call surveys: estimating site occupancy, detection probabilities and inferring absence. Biological Conservation, 123, 27-35.

Perissoratis C, Conispoliatis N (2003) The impacts of sea-level changes during latest Pleistocene and Holocene times on the morphology of the Ionian and Aegean seas (SE Alpine Europe). Marine Geology, 196, 145-156.

Petit RJ, Aguinagalde I, De Beaulieu JL et al. (2003) Glacial refugia: hotspots but not melting pots of genetic diversity. Science, 300, 1563-1565.

Posada D (2008) JModelTest: phylogenetic model averaging. Molecular Biology and Evolution, 25, 1253-1256.

Pritchard JK, Stephens M, Donnelly P (2000) Inference of population structure using multilocus genotype data. Genetics, 155, 945-959.

Provan J, Bennett KD (2008) Phylogeographic insights into cryptic glacial refugia. Trends in Ecology and Evolution, 23, 564-571.

RACE (2013) Risk assessment of Chytridiomycosis to European amphibian biodiversity. Available from: http://www. bd-maps.eu/
Ramos-Onsins SE, Rozas J (2002) Statistical properties of new neutrality tests against population growth. Molecular Biology and Evolution, 19, 2092-2100.

Reed DH, Frankham R (2003) Correlation between fitness and genetic diversity. Conservation Biology, 17, 230-237.

Reilly SB, Marks SB, Jennings WB (2012) Defining evolutionary boundaries across parapatric ecomorphs of Black Salamanders (Aneides flavipuntctatus) with conservation implications. Molecular Ecology, 21, 5745-5761.

Rosenberg NA (2004) Distruct: a program for the graphical display of population structure. Molecular Ecology Notes, 4, 137-138.

Rowe G, Beebee TJC (2003) Population on the verge of a mutational meltdown? Fitness costs of inbreeding load for an amphibian in the wild. Evolution, 57, 177-181.

Rowe G, Beebee TJC (2005) Intraspecific competition disadvantages inbred natterjack toad (Bufo calamita) genotypes over outbred ones in a shared pond environment. Journal of Animal Ecology, 74, 71-76.

Rowe G, Beebee TJC, Burke T (1999) Microsatellite heterozygosity, fitness and demography in natterjack toads Bufo calamita. Animal Conservation, 2, 85-92.

Rowe G, Harris DJ, Beebee TJC (2006) Lusitania revisited: a phylogeographic analysis of the natterjack toad Bufo calamita across its entire biogeographical range. Molecular Phylogenetics and Evolution, 39, 335-346.

Saccheri I, Kuussaari M, Kankare M et al. (1998) Inbreeding and extinction in a butterfly metapopulation. Nature, 392, 491-494.

Schmitt T (2007) Molecular biogeography of Europe: pleistocene cycles and postglacial trends. Frontiers in Zoology, 4, 13.

Schmitt T, Hewitt GM (2004) The genetic pattern of population threat and loss: a case study of butterflies. Molecular Ecology, 13, 21-31.

Schneider S, Excoffier L (1999) Estimation of demographic parameters from the distribution of pairwise differences when the mutation rates vary among sites: application to human mitochondrial DNA. Genetics, 152, 1079-1089.

Smith SA, Stephens PR, Wiens JJ (2005) Replicate patterns of species richness, historical biogeography and phylogeny in Holarctic tree frogs. Evolution, 59, 2433-2450.

Sotiropoulos K, Eleftherakos K, Džukić G et al. (2007) Phylogeny and biogeography of the alpine newt Mesotriton alpestris (Salamandridae, Caudata) inferred from mtDNA sequences. Molecular Phylogenetics and Evolution, 45, 211-226.

Stöck M, Sicilia A, Belfiore NM et al. (2008a) Post-Messinian evolutionary relationships across the Sicilian channel: mitochondrial and nuclear markers link a new green toad from Sicily to African relatives. BMC Evolutionary Biology, 8, 19.

Stöck M, Dubey S, Klütsch C et al. (2008b) Mitochondrial and nuclear phylogeny of circum-Mediterranean tree frogs from the Hyla arborea group. Molecular Phylogenetics and Evolution, 49, 1019-1024.

Stöck M, Dufresnes C, Litvinchuk SN et al. (2012) Cryptic diversity among Western Palearctic tree frogs: postglacial range expansion, range limits, and secondary contacts of three European tree frog lineages (Hyla arborea group). Molecular Phylogenetics and Evolution, 65, 1-9.

Suchard M, Lemey P (2013) Phylogeographic inference in continuous space. A hands-on practical. Version July 2013. Available from: http://beast.bio.ed.ac.uk/Tutorials 
Taberlet P, Cheddadi R (2002) Quaternary refugia and persistence of biodiversity. Science, 297, 2009-2010.

Ursenbacher S, Schweiger S, Tomović L et al. (2008) Molecular phylogeography of the nose-horned viper (Vipera ammodytes, Linnaeus (1758)): evidence for high genetic diversity and multiple refugia in the Balkan Peninsula. Molecular Phylogenetics and Evolution, 46, 116-1128.

Vitousek PM, Mooney HA, Lubchenco J et al. (1997) Human domination of Earth's ecosystems. Science, 277, 494-499.

Vos CC, Ter Braak CJF, Nieuwenhuizen W (2000) Incidence function modelling and conservation of the tree frog Hyla arborea in the Netherlands. Ecological Bulletin, 48, 165-180.

Waples RS, Gaggiotti O (2006) What is a population? An empirical evaluation of some genetic methods for identifying the number of gene pools and their degree of connectivity. Molecular Ecology, 15, 1419-1439.

Westemeier RL, Brawn JD, Simpson SA et al. (1998) Tracking the long-term decline and recovery of an isolated population. Science, 282, 1695-1698.

C.D. and N.P. designed research. C.D., J.W., K.G., M.St., P.L. and J.C.I. performed research. C.D., M.St. and A.B. contributed new markers. C.D. analysed data and drafted the manuscript, which was improved by N.P., A.B. and M.St.

\section{Data accessibility}

Sequences were deposited on GENBAnK, and Accession nos. listed in Table S3 (Supporting information). Microsatellite genotypes and sequences alignments were archived on Dryad (doi:10.5061/dryad.2vk30). Sequences of new microsatellite markers are available on GENBANK (Accession nos. can be found in Table S4, Supporting information).

\section{Supporting information}

Additional supporting information may be found in the online version of this article.
Data S1 Laboratory protocols for the amplification of the markers used in this study.

Data S2 Phylogeographic reconstruction in continuous space using relaxed-random walks (Lognormal), based on mitochondrial sequences.

Fig. S1 Conservation status of Hyla arborea on national and regional red lists, when available.

Fig. S2 Maximum-Likelihood phylogeny of 112 mitochondrial haplotypes (concatenated D-loop and cyt b).

Fig. S3 Maximum-Likelihood phylogeny of 27 haplotypes from the nuclear rag-1.

Fig. S4 Likelihood probability of the data $L(K)$ and $\Delta K$ ad hoc statistics computed from Structure runs with Structure HarVESTER (ten replicates per $K$ ).

Fig. S5 Principal Component (PCA) of microsatellite allelic frequencies.

Fig. S6 Intergroups gene flow $x \mathrm{~N}_{\mathrm{e}} \mathrm{m}$ estimated by Migrate (first values: from sequence data / second values: from microsatellite data).

Table S1 National and regional red lists assessments of Hyla arborea, and population trends.

Table S2 Information on the localities included in this study, and their genetic diversity.

Table S3 GenBank Accession nos of cytochrome b, D-loop and rag- 1 sequences, and availability of microsatellite genotypes.

Table S4 Details on the genetic markers used in this study.

Table S5 Log marginal likelihood estimated from path (PS) and stepping stone (SS) sampling from mtDNA Bayesian inferences under different continuous spatial diffusion models.

Table S6 Demographic parameters $\theta$ and $M$, estimated with Migrate from sequences and microsatellites. 95\% confidence intervals are given in brackets. 